\title{
Información del PH \\ Las catedrales andaluzas. Dinámicas de los espacios urbanos
}

Pedro Salmerón Escobar

Arquitecto

\section{Resumen}

Las catedrales andaluzas pueden colaborar activamente en la revitalización de los centros históricos de las ciudades actuando como impulsoras de la revitalización fisica y funcional de sus entornos urbanos, ayudando a plantear nuevas orientaciones para disminuir la contaminación ambiental y el tráfico rodado y conseguir una mayor protagonismo del peatón en las áreas centrales.

Los flujos de visitantes hacia las catedrales y otros monumentos y enclaves del centro de la ciudad deben estimular politicas urbanas que supongan mejoras cualitativas de habitación, comercio, hosteleria y usos culturales sin fomentar la aparición de "monocultivos turisticos".

\section{Palabras clave}

\section{Catedrales}

Plan de Catedrales

Andalucia

Centros históricos

Planes directores de catedrales

Patrimonio inmueble

Tráfico urbano

Contaminación

\section{El primer Plan de Catedrales de} Andalucía

La Consejería de Cultura de la Junta de Andalucía proyectó en 1988 el Plan de Catedrales de Andalucía como plan marco en el que desarrollar las acciones sobre un conjunto de inmuebles de gran calado en la región. Las trece catedrales podian convertirse, de alguna forma, en el modelo de otras intervenciones similares en el patrimonio andaluz y en ese aspecto el Plan se perfilaba como pionero en la política de los bienes culturales en el país. Con independencia de las acciones que hubiesen emprendido el Ministerio de Cultura o los Cabildos Catedralicios, la Consejería de Cultura ejercía su papel como administración competente en un aspecto fundamental del patrimonio cultural, y abría la participación a los propios cabildos a través de la Comisión Mixta correspondiente.

Aunque se solicitó de los equipos nombrados al efecto la formulación de los trabajos más urgentes que debían acometerse, el Plan de Catedrales de Andalucia iba más allá de estas acciones puntuales; en realidad planteaba una acción combinada en varios campos que se resumía en tres grandes apartados:

Plan de Catedrales de Andalucía (1988)

Apartados básicos para el desarrollo de los trabajos

$>$ Definición y ejecución de las intervenciones de emergencia

$>$ Definición de intervenciones ordinarias

$>$ Levantamiento fotogramétrico

$>$ Diagnóstico previo

En aquel momento ya se consideraba de interés ofrecer un planteamiento que trascendiera al objeto e implicase a la propia ciudad porque las catedrales forman parte esencial de los cascos históricos, y el deterioro, como proceso lógico de envejecimiento y transformación de las estructuras físicas, se convierte en un recorrido de influencias mutuas, donde la decadencia o la vitalidad de la ciudad y sus gentes tiene un reflejo y respuesta incuestionables en estos grandes edificios ${ }^{1}$.

Se trataba de considerar la relación ambiente - ciudad - monumento que nos hace pensar en los problemas de tipo climático, biológico, etc. que influyen decisivamente sobre la conservación, pero también era la ocasión de iniciar los estudios de las transformaciones que imponen otros factores de un calado nada despreciable a medio y largo plazo, como el uso turístico y el papel añadido de centralidad de los ámbitos inmediatos a las catedrales que pueden ejercer una función revitalizadora de los centros históricos.

Hoy deberiamos profundizar de manera intensa en estas relaciones porque las catedrales están en el centro de muchas acciones de revitalización, de uso de los recursos patrimoniales, de intensificación de la demanda turística, etc. 


\section{$054-055$ \\ Información del PH}

Las catedrales andaluzas. Dinámicas de los espacios urbanos

\section{PH47 - Febrero 2004}

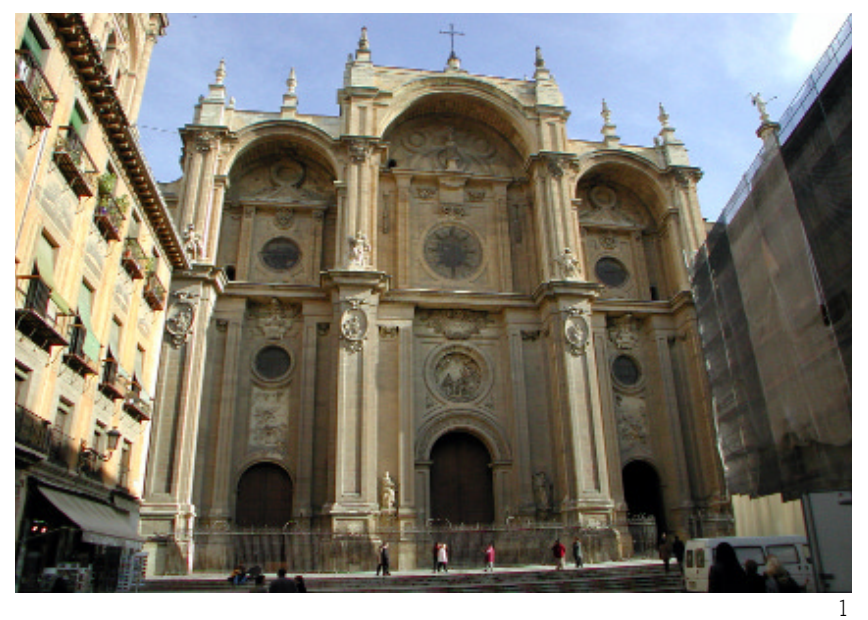

\section{Acotaciones a la formación de algunos espacios urbanos en relación con las catedrales}

Las catedrales españolas se construyen a través de un arco temporal muy amplio. La concepción medieval del templo catedralicio pertenece sin lugar a dudas a Europa Central, aunque es en España donde se produce el cierre de esta concepción con dos ejemplos especialmente significativos e interesentes, las catedrales tardogóticas de Salamanca y Segovia ${ }^{2}$, cuya construcción finaliza en el s. XVI. Pero en nuestro caso tiene lugar un hecho tanto o más trascendente que el de su nacimiento: nos referimos a la finalización del proceso de construcción de las catedrales en el Renacimiento y a su culminación en el barroco.

En las catedrales andaluzas el barroco tiene un peso decisivo en los casos de Granada, Jaén y Guadix, con fórmulas expresivas que, usadas como contrapunto de evidente maestría, cierran los programas renacentistas originales. 0 la nueva de Cádiz debida al arquitecto barroco Vicente Acero que se termina en estilo neoclásico.

Las catedrales son una parte muy activa, cuando no central, en la formación de la ciudad histórica. Las andaluzas son, en gran parte de los casos, piezas esenciales del enlace con una etapa histórica prolongada, la hispanomusulmana, en la que muchas de las capitales de Andalucia alcanzaron una madurez importante como organismos complejos, profusamente habitados. Las catedrales propulsan un plano de actividad de gran interés, vinculado a las nuevas concepciones del centro urbano y a la función religiosa, añadida como un factor de dominio de la ciudad a través de las iglesias parroquiales, que aprovechan en muchas ocasiones el emplazamiento de antiguas mezquitas, en una posición refleja del mecanismo de producción de la propia catedral. O los conventos, que se sitúan estratégicamente en una reinvención de las relaciones centro - periferia, véase el caso de Sevilla con una cantidad inusitada de conventos instalados desde el s. XIII al s. XVIII tanto en el
1. Catedral de Granada. Fachada de Alonso Cano hacia la plaza de las Pasiegas como cierre del programa arquitectónico iniciado en el siglo XVI

2. Catedral de Sevilla y Archivo de Indias en el frente de Avda. de la Constitución que se abre progresivamente hacia las Atarazanas, el Hospital de la Caridad, la Casa de la Moneda y el río Guadalquivir

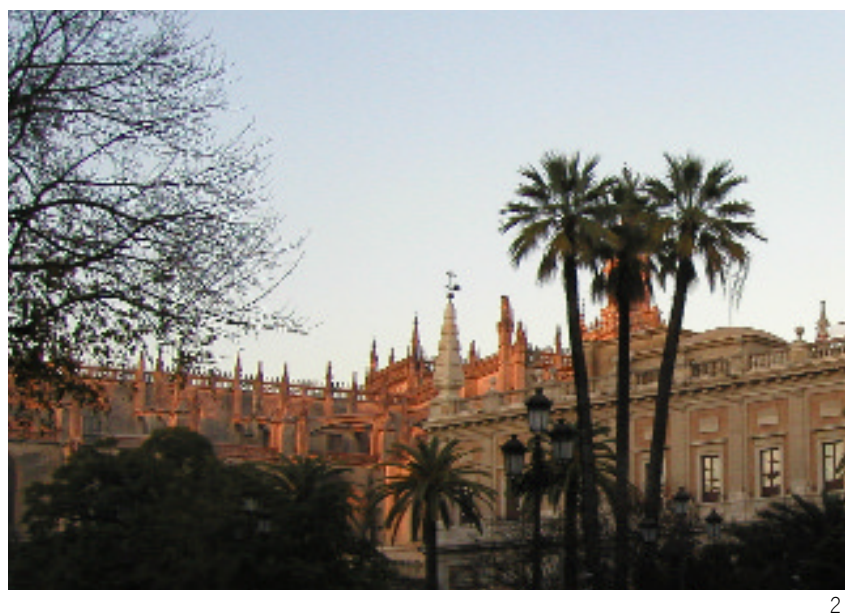

interior del recinto amurallado como en la periferia, con casos tan significativos como La Cartuja, Trinidad o San Agustín ${ }^{3}$.

La ocupación de las mezquitas aljamas de algunas ciudades andaluzas por los grandes templos catedralicios supone en sí misma una premisa para la reactivación de los centros neurálgicos de estas capitales, y un ejemplo de lo que ocurre en otros casos de menor envergadura, que en cualquier caso convendría analizar en un estudio de mayor profundidad que éste.

En Sevilla, la mezquita aljama almohade, situada junto a la alcaicería y los alcázares, ocupa una posición excéntrica en la trama urbana cerrada hasta el s. XII por las murallas almorávides y almohades. Lo decisivo de esta ubicación está en su posición privilegiada respecto al río Guadalquivir y al contacto con un eje excepcional de relaciones que lleva hasta el puerto fluvial y se extiende a lo largo del tiempo con instalaciones tan importantes como las Atarazanas, la Lonja de los Genoveses, la Aduana, la Casa de la Moneda, la Fábrica de Tabacos, etc., y se completa hacia el "interior" con la Lonja de los Catalanes, la Lonja de Placentines, el Ayuntamiento... La formación del templo catedralicio sobre la mezquita, con una planta de gran extensión y un programa que se cierra magistralmente en el Renacimiento define de manera temprana las relaciones urbanas y su protagonismo formal y simbólico en el frente portuario, cuestión que queda expresada con claridad en muchas vistas de Sevilla.

En Granada se había conformado un eje lineal de edificaciones según la orientación SO-NE que empezaba por la antigua mezquita aljama, en la que se había instalado, sin apenas transformaciones, el primer templo de advocación cristiana con el nombre de Sta. María de la $\mathrm{O}^{4}$. Esta implantación se completó con la Lonja y la Capilla Real. Este flanco de escala inusitada, cuya referencia primordial era el río Darro, estaba apoyado en un punto vital y especialmente estructurado de la trama urbana medieval, colocado en paralelo al Zacatín y sólidamente acompañado por el Mercado de la Seda (Alcaicería) y la Universidad de los Príncipes (Madraza). A partir de ahi el nuevo orden urbano se abría con una demanda im- 
3. La catedral de Málaga. Vista desde el puerto hacia el frente urbano de la plaza de la Marina

4. La catedral de Málaga desde la calle San Agustín, eje de gran actividad gracias al nuevo Museo Picasso

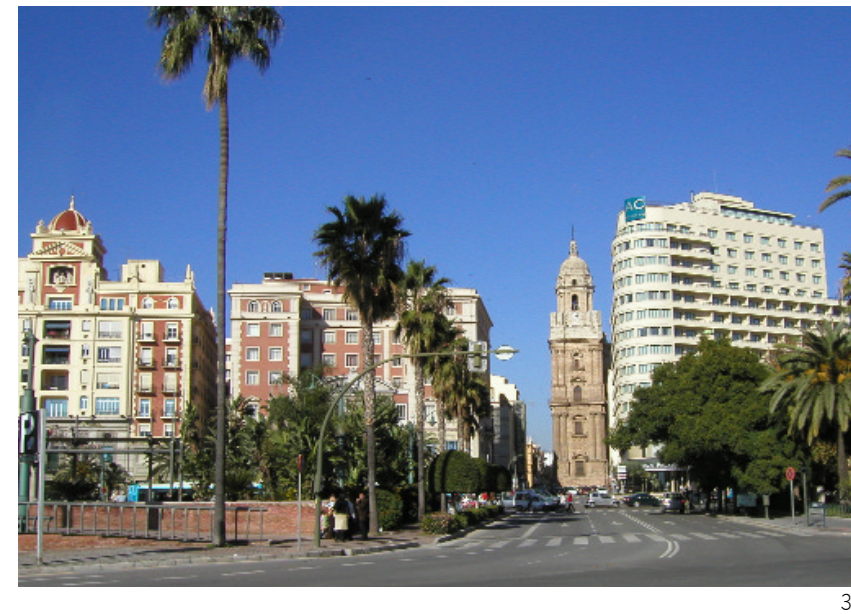

portante de suelo hacia el NO con la portada principal de la CapiIla Real, que poco más tarde quedaría englobada hacia el interior del transepto de la Catedral. El gran solar de la instalación catedralicia quedó marcado por el eje al que nos hemos referido ganando todo el espacio necesario a costa de la trama urbana preexistente, definiendo finalmente una decisiva alineación hacia la actual calle Cárcel $^{5}$ y un cierre que culmina en el barroco hacia la plaza de las Pasiegas con el proyecto de fachada de Alonso Cano lleno de sugerencias espaciales y volumétricas.

La catedral granadina aprovecha la calidad de las relaciones establecidas en la ciudad hispanomusulmana, produciendo un vaciado e inmediata ocupación de una parte importante de la trama medieval y manteniendo la constricción de los espacios periféricos. Algo relativamente frecuente en los templos catedralicios cuya fundación sobre tramas preexistentes, ocupadas y vivas, impide una verdadera ampliación de los espacios de observación y de relación que, en la mayor parte de los casos, se materializan en una comedida dilatación del vacío urbano frente a la fachada principal. No es posible comparar este procedimiento de implantación y desarrollo con otros casos de ciudades europeas que tuvieron una capacidad mucho mayor de transformación de la trama urbana. Tengamos en cuenta que la operación de conquista de los puntos más significativos de Granada a partir del programa religioso y civil establecido por los Reyes Católicos a su llegada a la ciudad, con los hospitales, las iglesias parroquiales, los conventos, la Audiencia, etc. hizo que la ciudad fuese tomada paulatinamente sin ampliaciones de trascendencia del espacio público. Las claves de la Granada baja se marcaron en el siglo XVI y sirvieron de pauta para desarrollos posteriores. Aunque en el siglo XV la Granada nazari es ya una ciudad extendida hacia la llanura, con plazas y alhóndigas localizadas junto a la muralla inferior, es la Granada cristiana la que rompe definitivamente con la muralla defensiva, primero abriendo huecos entre los que se da continuidad a la trama urbana preexistente, después derribando lienzos completos de la muralla baja, que en algunos casos queda simbolizada por puertas y bastiones que también van cayendo a lo largo de un proceso que dura cuatro siglos, del que hay que ex-

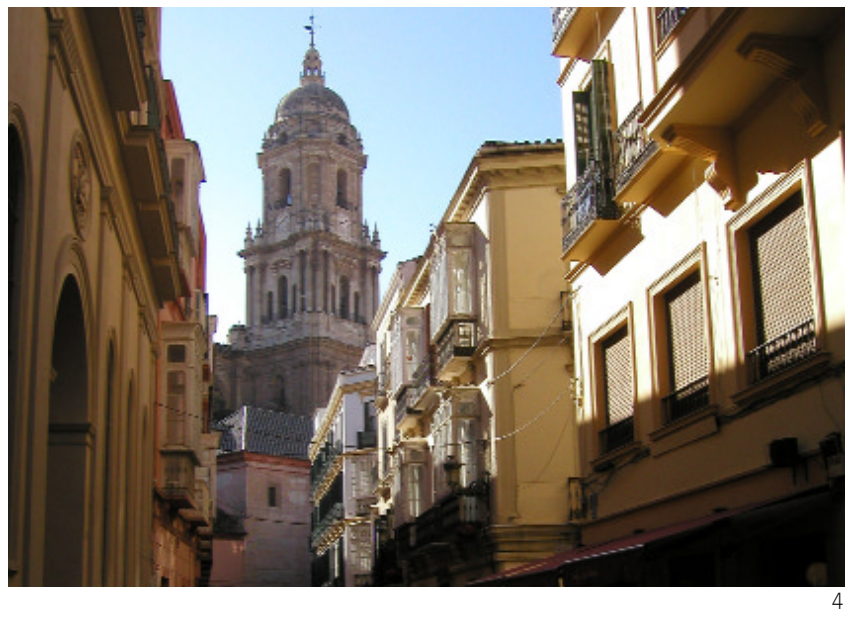

ceptuar la puerta de Elvira que permanece como espléndido símbolo de los accesos de la muralla en la ciudad baja.

\section{Catedrales y dinámicas de los centros históricos}

Con el propósito limitado de este artículo, pero otorgándole la posibilidad de colaborar en el inicio de una reflexión necesaria, resulta conveniente analizar algunas coordenadas de la situación actual de las catedrales andaluzas, que tienen una andadura importante en el terreno de la conservación y menos perspectiva en el plano de las relaciones urbanas, con la salvedad de la intensidad de intercambios que se establecen a partir de la visita turística. Cuestión especialmente relevante en Sevilla con una conexión singular con los Reales Alcázares, en Córdoba por el papel excepcional de la mezquita - catedral, o en Granada por el atractivo de la Capilla Real con el enterramiento de los Reyes Católicos (en torno a 700.000 visitas), ya que actúa como verdadero puente con los visitantes de la Alhambra (más de 2 millones al año). A su vez la catedral representa un factor de atracción en ciudades como Málaga con un sector turístico muy desarrollado (Costa del Sol) que visita la ciudad aunque sea con otras perspectivas, ahora reforzadas con el Museo Picasso conectado con la catedral a través de la calle San Agustín. 0 en Jaén, con un importante número de visitas de carácter "interno" vinculadas al culto o a la devoción, y otras de grupos que se desplazan en autobús aprovechando la buena posición de la ciudad en los recorridos que se establecen desde Madrid.

Las transformaciones, que se han ido produciendo en las ciudades a partir del turismo, son expresivas de la capacidad de generar actividad por la presencia de un número significativo de visitantes que se introducen en los circulos de consumo de la ciudad, especialmente los comercios y las instalaciones relacionadas con la hostelería y la restauración: hoteles, bares, cafeterías, etc. La posibilidad de que esta interacción esté articulada depende de medidas que generalmente no se toman y no se produce una extensión de la visita a otros lugares de la ciudad o el entorno, o una 


\section{$056-057$ \\ Información del PH}

Las catedrales andaluzas.

Dinámicas de los espacios urbanos
5. Relaciones de la Alhambra y el centro de Granada que pueden verse favorecidas con un protagonismo mayor de la Catedral, Capilla Real, Madraza, Corral del Carbón etc. y la propia trama urbana. Dibujado a partir del Estudio Previo para la revisión del Plan Especial de la Alhambra y Generalife. M. A. Troitiño y cols. Patronato de la Alhambra y Generalife. Granada, 1999

\section{PH47 - Febrero 2004}

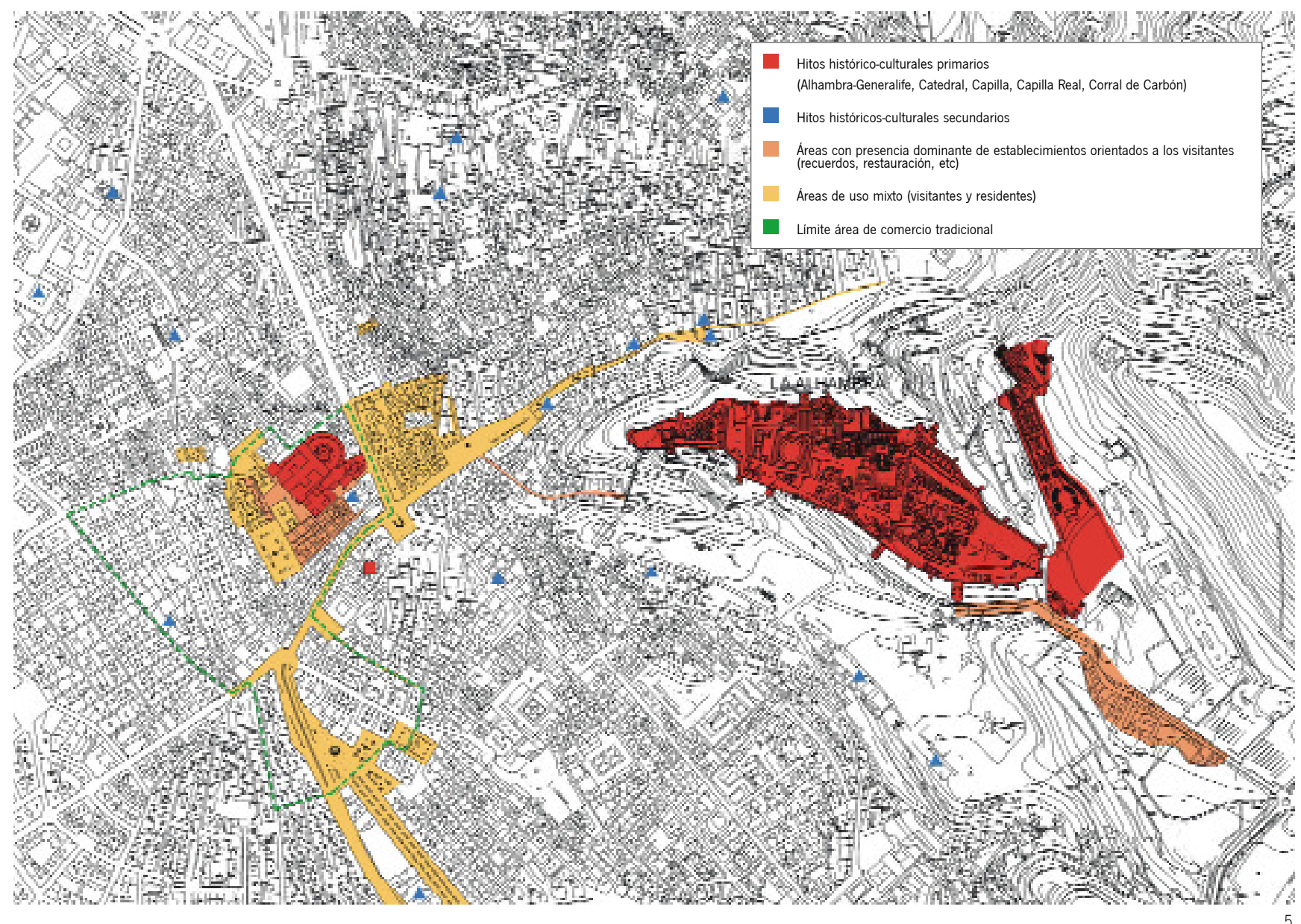

prolongación de la estancia del visitante, salvo ciudades que han logrado establecer una oferta mayor basada en un patrimonio abundante 0 en otras actividades culturales asociadas aspecto que se cumple, al menos parcialmente, en Sevilla, Granada o Córdoba.

En casos paradigmáticos de esta relación compleja turismo - ciudad, como es el caso de Granada, es conveniente advertir sobre problemas estructurales que nos hablan de la dificultad de establecer planes de revitalización de las áreas centrales de las ciudades, incluso desde perspectivas como la del turismo, que se acepta como de interés general en algunos enclaves de los que estamos tratando. En Granada, 69 BIC, dos conjuntos históricos, el de Granada y el de la Alhambra -ahora rectificada su calificación como Monumento-, 573 edificios de interés y otros 564 de interés ambiental censados por el planeamiento urbanístico, con dos piezas fundamentales de su estructura urbana, la Alhambra y el Albaicín, incluidas en la Lista de Patrimonio Mundial, y una oferta cultural de considerable magnitud no logran cambiar la visita turística, que se desenvuelve entre unos pocos hitos monumentales y el tránsito por el centro histórico por parte de aquellos visitantes que comparten la Alhambra con la ciudad (situación cada vez más frecuente). La focalización excesiva de la visi- ta en la Alhambra que comparte a distancia la Capilla Real - Catedral, favorece el deterioro del patrimonio, la aparición de fenómenos de "monocultivo turístico" y la banalización de la visita y compromete la propia sostenibilidad del proceso ${ }^{6}$.

Esta reflexión podría trasladarse, aunque con otras coordenadas muy diferentes, a otros monumentos que están en el centro del atractivo turístico de nuestras ciudades, encontrándose las catedrales en el núcleo principal de ese proceso de transformación en el que la intensidad del uso turístico sin una respuesta coordinada de la propia ciudad, produce un aumento de la situación de deterioro y de falta de sostenibilidad "corredor turístico" de Toledo, zonas inmediatas a la catedral de Granada con puestos de souvenir en la misma calle Oficios y Estribo, etc.).

En estas circunstancias resulta especialmente interesante una primera reflexión que conecta con los entornos de los monumentos, entendidos como un medio para impulsar las relaciones de éstos con su contexto para el desarrollo social, cultural y productivo ${ }^{7}$ del mismo en un marco sostenible y respetuoso con sus valores, y capaz de potenciar un entendimiento y disfrute del monumento desde perspectivas que no conviertan al visitante en 
6. Propuesta de entorno del historiador del arte José Castillo Ruiz para la Catedral de Granada y su relación con otros edificios de interés del Centro Histórico.

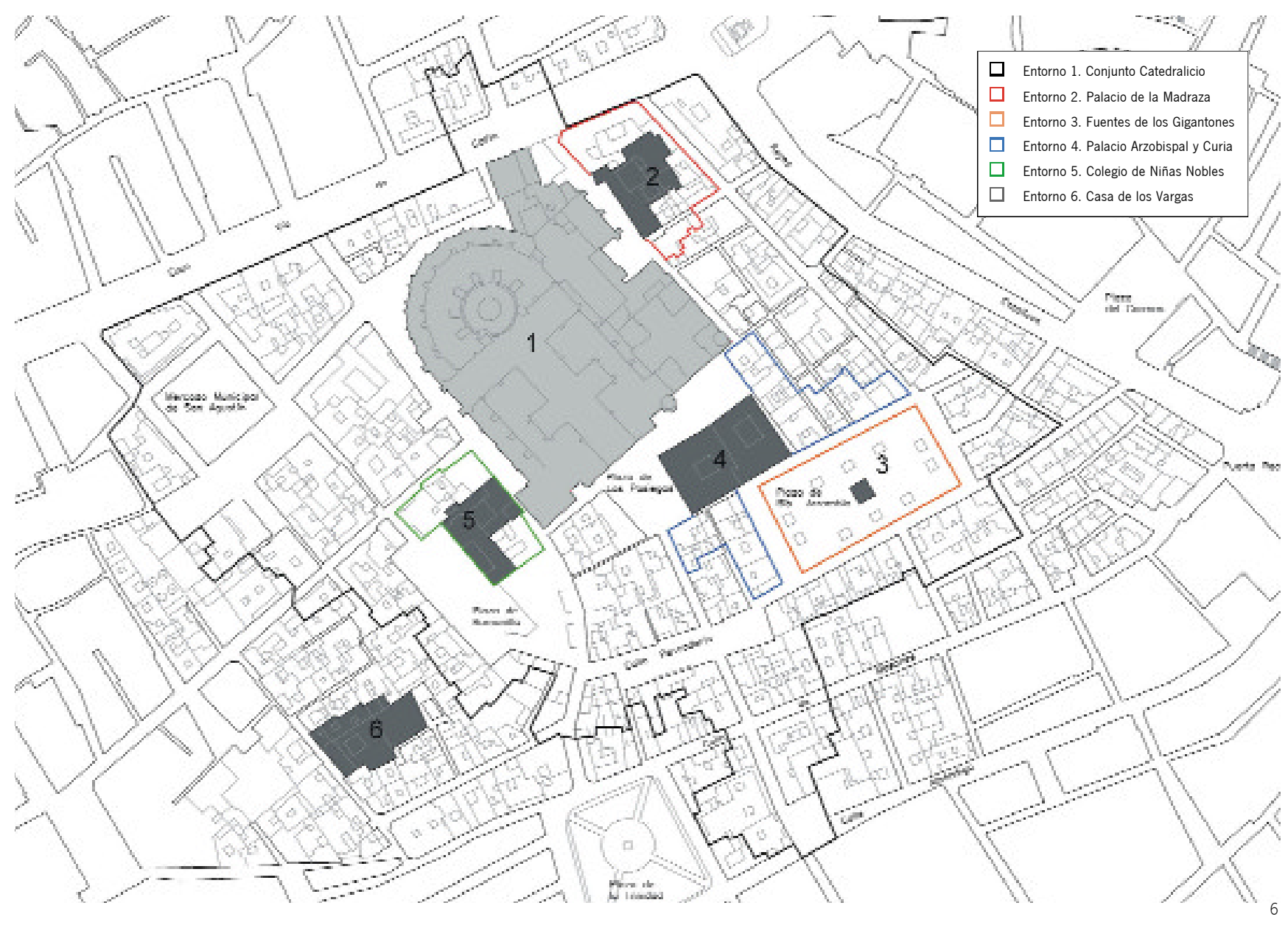

sujeto pasivo. Aunque por razones de economía de medios y de prudencia con respecto a la gestión urbanística de los centros históricos, los entornos se reduzcan a una dimensión cercana al monumento, su papel no deja de tener un interés notable como punto de confluencia de las acciones posibles de las administraciones y de los agentes privados. La relación entre los entornos catedralicios y la ordenación de los centros históricos podría entenderse en esa línea y se debe ensayar la definición de los entornos, ya que se produce la paradoja de su inexistencia a pesar de la importancia de estos inmuebles. La situación actual permite afirmar que sólo una catedral, la Catedral Nueva de Cádiz, tiene entorno delimitado; el resto carece de tal definición. Se adjunta un listado ${ }^{8}$ de las catedrales andaluzas y la fecha de su declaración como BIC que influye decisivamente en la carencia de esta definición, ya que en su mayoría se produjo durante la Segunda República, momento en el que fue suficiente la publicación del nombre el monumento en la Gaceta de Madrid.

En estos momentos se presenta otra circunstancia de interés: los planes directores de las catedrales andaluzas se han redactado sustituyendo al primer Plan de Catedrales Andaluzas de 1988 que especificaba una serie de medidas de conjunto. En la ac- tualidad las diferentes catedrales tienen un plan director especifico como primer desarrollo (2000) en el que se centran los aspectos más importantes y se programan las acciones sobre las mismas. Están en muchos casos pendientes del acuerdo formal entre la Consejería de Cultura, el Ministerio de Cultura y los cabildos y obispados de las diferentes diócesis, pero en la práctica están orientando las intervenciones que se vienen realizando en los últimos años. Es la ocasión para establecer un programa que tenga una cierta dimensión urbana y permita unas perspectivas novedosas en una relación que, bien planificada y orientada, puede dar excelentes resultados.

En algunos casos las necesidades de una mejor presentación de una parte del patrimonio catedralicio podría implicar a otros edificios de la zona, dándose una expansión de gran interés de los límites de las catedrales. Puede servir de ejemplo el de la Catedral de Málaga, donde la necesidad de nuevos espacios museísticos o de archivo podría resolverse en el entorno próximo, incorporando al programa de uso y gestión de la catedral inmuebles cercanos como el Hospital de Santo Tomás -edificio decimonónico de interés monumental, sin uso en la actualidad, frente a la iglesia del Sagrario-. Esto requiere estudiar también otras alter- 


\section{$058-059$ \\ Información del PH}

Las catedrales andaluzas.

Dinámicas de los espacios

urbanos

\section{PH47 - Febrero 2004}

7. La Catedral de Sevilla soporta deterioros importantes debidos al tráfico urbano, especialmente intenso en Avda. de la Constitución

8. La supresión del tráfico rodado en la calle Molina Lario, en el tramo de la plaza del Obispo, sería un factor decisivo para la contemplación de la Catedral de Málaga y para facilitar los flujos peatonales hacia todo el entorno catedralicio y el eje del Museo Picasso

9. La Carrera de Jesús. Un eje que prolonga las visuales de la Catedral de Jaén y dispone de edificios de interés que pueden recuperarse

10. Tráfico intenso en las calles estrechas y sugerentes que bordean la catedral de Jaén

Los entornos de las catedrales andaluzas

\section{Catedral}

Almería. Iglesia Catedral de la Encarnación

Cádiz. Catedral Nueva

Cádiz. Catedral Vieja

Cádiz. Jerez de la Frontera

Córdoba. Mezquita Mayor - Iglesia Catedral de la Asunción

Granada. Iglesia Catedral de la Encarnación

Granada. Baza. Iglesia Mayor Concatedral de la Anunciación

Granada. Guadix. Iglesia Catedral de la Encarnación

Huelva. Iglesia Catedral de Ntra. Sra. de la Merced

Jaén. Iglesia Catedral de la Asunción

Jaén. Baeza. Iglesia Catedral de la Natividad

Málaga. Iglesia Catedral de la Encarnación

Sevilla. Iglesia Catedral de Santa María
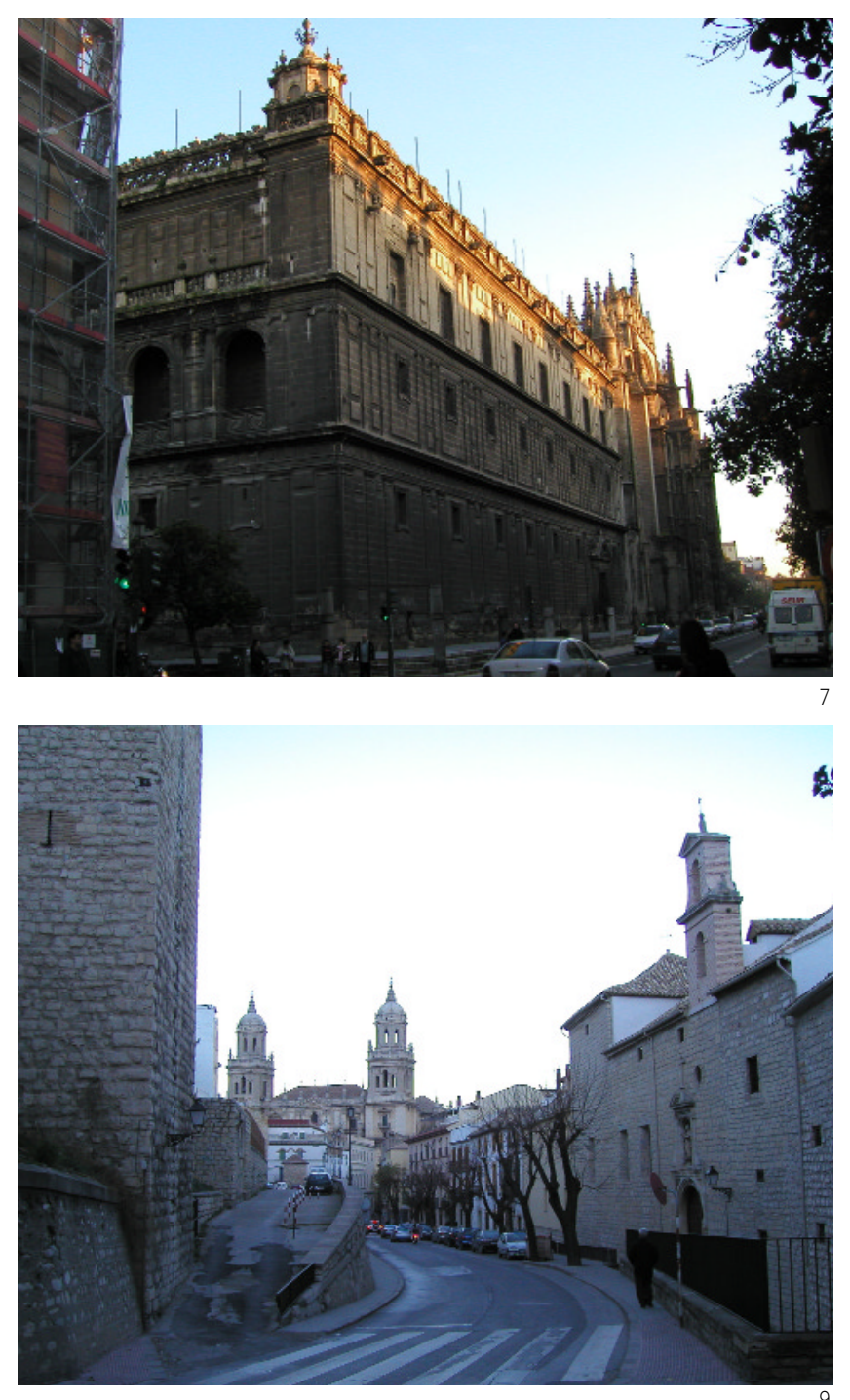

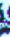

\section{Fecha declaración}

03/06/1931

03/06/1931

10/03/1987 (incoación)

03/06/1931

21/11/1882

02/11/1929

03/06/1931

03/06/1931

12/03/1970

03/06/1931

03/06/1931

03/06/1931

29/12/1928
Entorno

$28 / 11 / 2000$
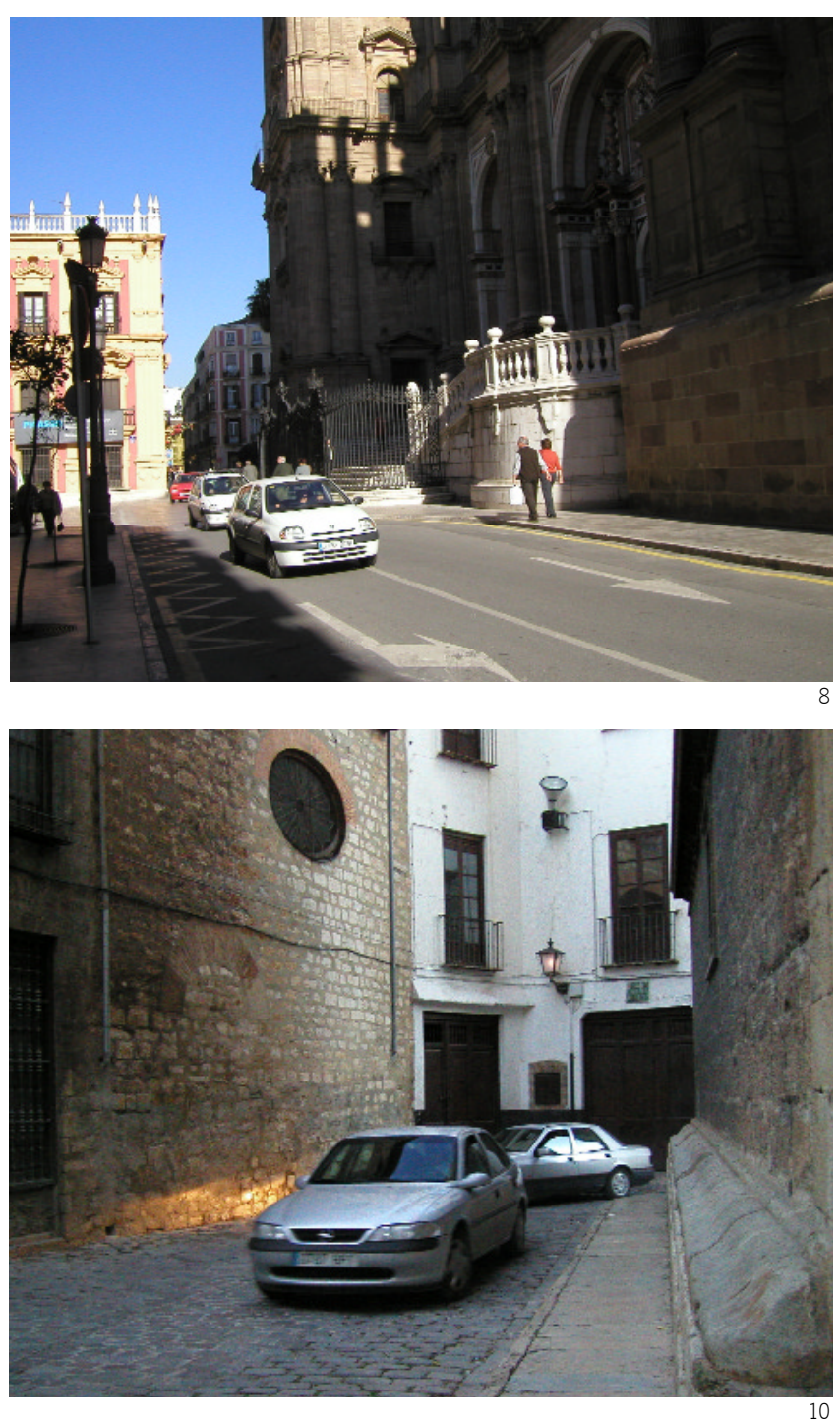
11. Vista de la calle Abades junto a la Catedral de Jaén, una zona urbana situada en el frente sur cuya revitalización permitiria recuperar los valores de centralidad urbana del entorno catedralicio

nativas, pero en definitiva la propuesta supondría empezar a considerar de forma clara la dimensión urbana de las catedrales evitando la sobrecarga de nuevos usos en el interior de los templos, cuando se quiere mejorar la presentación de ese patrimonio hacia el exterior ${ }^{9}$.

Cuestiones tan importantes como la ordenación del tráfico es posible que puedan plantearse en esta escala urbana del problema, véase el caso de Granada con la calle Cárcel que añade una problemática preocupante a la conservación del monumento, o en Málaga la posible restricción general del tráfico circundante, la eliminación de los aparcamientos que contaminan la percepción visual inmediata del monumento en calle Cister y la parada de autobuses en Postigos de los Abades, y la peatonalización de la calle Molina Lario en el tramo de la Plaza del Obispo. En Sevilla, los problemas acuciantes de la catedral en relación con el tráfico hacen imposible un planteamiento a medio plazo de la restauración de la piedra en buena parte del monumento.

En el entorno cercano a la Catedral de Jaén interesa destacar, porque está lleno de posibilidades y pasa desapercibido para el visitante: la calle Carrera de Jesús (Juan Montilla) que contiene varios edificios notables (algunos arruinados como el antiguo Diario Jaén, frente al Ayuntamiento), casonas de gran interés como el Antiguo Bar Conde Duque que antes fue sede de la Caja de Ahorros de Córdoba, el Camarín de Jesús (actualmente en recuperación como unidad de ejecución del Plan Especial) y el Torreón del Conde de Torralba y parte de la muralla de Jaén (el Torreón actualmente sólo es accesible por el Colegio de Santo Tomás, con entrada en Plaza Cruz Conde). Bajo la Muralla, conservada como traseras de la calle del Obispo existen unos locales que serían ideales para establecer talleres de artesanías y un mercado. A esta relación se puede sumar el edificio del Banco de España, ahora ocupado por el Catastro, que podría servir como espacio museístico asociado a la catedral. Hay que considerar las vistas que desde este edifico hay hacia la logia Sur de la Catedral, que serían un valor añadido a un uso cultural de este inmueble.

Hasta ahora los grupos que visitan la Catedral se centran en el interior del edificio y en su fachada y sería interesante valorar un recorrido por el perímetro, en especial el lado Sur, que es una zona de tráfico dificultoso. Peatonalizar y regenerar esta zona sería del máximo interés porque el barrio inmediato -calles Llana, Abades, Espiga- conserva todo el sabor aunque las sustituciones son cada vez más frecuentes ${ }^{10}$.

Estos ejemplos nos sirven de pauta para iniciar un planteamiento de doble recuperación en el que se verian implicados los centros históricos en estas zonas centrales de su trama, que tienen como grandes protagonistas a las catedrales andaluzas, ejemplos de arquitecturas que son ciudad en sí mismas y se presentan con un futuro excelente para este papel mediador con la cultura y el patrimonio urbanos.

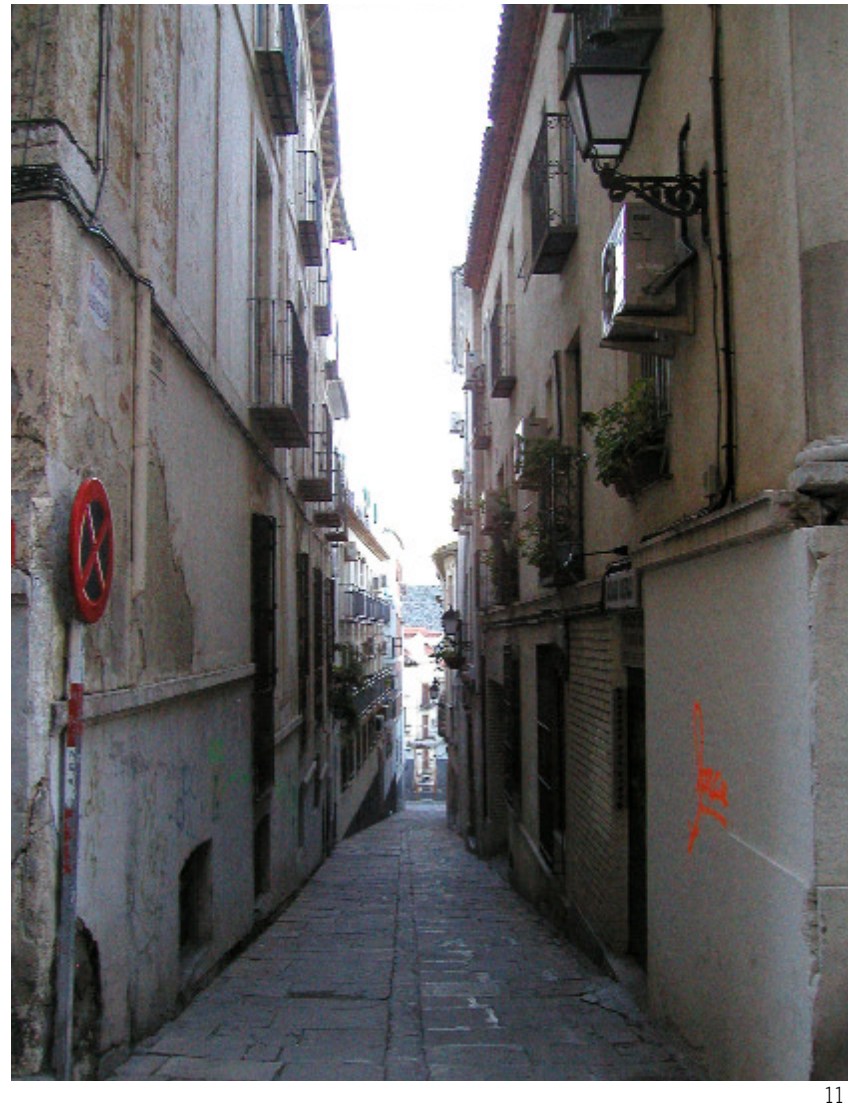

${ }^{1}$ Véase texto del autor: "El papel de la analítica de los materiales en la restauración del patrimonio inmueble" en Metodologia del diagnóstico y evaluación de tratamientos para la conservación de los edificios históricos. Colección Cuadernos Técnicos. Sevilla, Instituto Andaluz del Patrimonio, 2003

${ }^{2}$ Véase Carlos SARTHOU CARRERES y Pedro NAVASCUÉS PALACIO. Catedrales de España. Madrid, 1988.

${ }^{3}$ La instalación de los conventos en el casco antiguo de Sevilla puede verse representada con claridad en el Atlas Histórico de ciudades europeas. AA.W. -Península Ibérica-. Barcelona, 1994.

${ }^{4}$ En ese emplazamiento se construye más tarde un bello edificio barroco, la iglesia parroquial del Sagrario.

${ }^{5}$ Cf. Pedro SALMERÓN. La Catedral de Granada. Un proyecto entre dos visiones del espacio arquitectónico. Catálogo de la Exposición Jesucristo y el Emperador Cristiano. Granada, 2001.

${ }^{6}$ Son especialmente interesantes las observaciones del Estudio previo para la revisión del Plan Especial de la Alhambra y Alijares -Documento previo de sintesis y diagnóstico-. Miguel Ángel TROITIÑO VINUESA y cols. Granada, Patronato de la Alhambra y Generalife, 1999.

${ }^{7} \mathrm{Cf}$. por su especial interés en lo que respecta al papel de los entornos: José CASTILLO RUIZ. El entorno de los Bienes de Interés Cultural. Granada, IAPH / Universidad de Granada, 1977.

${ }^{8}$ Información obtenida del Servicio de Información del Centro de Documentación del Instituto Andaluz del Patrimonio Histórico.

${ }^{9}$ Información elaborada a partir de la colaboración solicitada para este tema a la historiadora María Morente.

${ }^{10}$ Información elaborada a partir de la colaboración prestada por la arqueóloga Francisca Hornos. 